\title{
Influence de la lumière et de la température sur la croissance de quatre espèces d'algues d'un lac eutrophe (Lac d'Aydat, Puy de Dôme, France)
}

\author{
L. Boumnich1 \\ A. Dauta ${ }^{2}$ \\ J. Devaux 1 \\ J.C. Romagoux ${ }^{1}$
}

Mots clés : phytoplancton, taux de croissance, lumière, température.

L.'interdépendance de la lumière et de la température est étudièe en milieu fini et en conditions nutritives non limitantes sur des cultures monospécifiques non axéniques de quatre espèces : Fragilaria crotonensis, Anabaena macrospora, Staurastrum pingue et Coelastrum cambricum. Les taux de croissance maximum sont respectivement de $0.57,0.63,0.77$ et 1.4.j-1 associés à des températures de $25,25,27$ et $35^{\circ} \mathrm{C}$ et des lumières optimales de $150,170,270$ et $290{ }_{\mu} \mathrm{E}^{-1} \mathrm{~m}^{-2} \mathrm{~s}^{-1}$. Ces résultats obtenus, dans une plage dè température $10-35^{\circ} \mathrm{C}$ et pour des lumières comprises entre 5 et $800 \mu \mathrm{E} . \mathrm{m}^{-2} . \mathrm{s}^{-1}$, sous une photopériode de $15 / 9$, sont ajustés par des modèles.

Influence of light and temperature on the growth of four algae from a eutrophic lake (Lake Aydat, Puy de Dôme, France) Keywords: phytoplankton, growth rate, light, temperature.

The interdependence between light and temperature was studied in batches and in non nutrient-limited conditions for single strain, non axenic cultures of four species : Fragilaria crotonensis, Anabaena macrospora, Staurastrum pingue and Coelastrum cambricum. The maximum growth rates were found to be $0.57 .0 .63,0.77$ and 1.4 day ${ }^{-1}$, respectively at temperatures of $25,25,27$ and $35^{\circ} \mathrm{C}$ and light intensities of $150,170,270$ and $290 \mu$ E.m ${ }^{-2} \cdot \mathrm{s}^{-1}$. Results were obtained over a temperature range of 10 to $35^{\circ} \mathrm{C}$ and light intensities between 5 and $800 \mu \mathrm{E} . \mathrm{m}^{-2}, \mathrm{~s}^{-1}$ under a $15 / 9$ photoperiod and were adjusted by a model.

\section{Introduction}

Au sein des écosystèmes limniques et océaniques, la biomasse phytoplanctonique se résume le plus souvent à la succession d'un nombre limité d'espèces dominantes qui constituent la fraction la plus productive, dont le développement peut être dû

1. Groupe de Zoologie et Protistologie, URA CNRS 138, Université Blaise-Pascal Clermont II,63177 Aubière Cedex, France.

2. Laboratoire d'Hydrobiologie, Univer sité Paul-Sabatier, UA 695 du CNRS, 118, route de Narbonne, 31062 Toulouse Cédex, France.

Etude réalisè dans le cadre du programme PIREN : Cycle biogéochimique du phosphore. soit à des facteurs physiques, chimiques et/ou biologiques (Reynolds 1984). Cependant il s'est avéré indispensable de ne plus considérer le peuplement phytoplanctonique comme une entité indivisible : diverses composantes peuvent être distinguées sur la base des caractéristiques physiologiques de chaque espèce selon leurs réponses aux changements de milieu (Canale et al. 1976, Bierman 1976).

Grand nombre de travaux consacrés à l'étude écophysiologique des algues, ont montré qu'il existe des interactions complexes entre lumièretempérature et les nutriments (Steeman-Nielsen \& Jorgensen 1968). Le taux instantané $(\mu)$ d'une 
population représente l'action de ces paramètres lorsqu'ils s'éloignent des valeurs optimales, affectant le taux maximal spécifique de croissance (Dauta 1982). Ceci peut être résumé dans l'expression suivante :

$$
\mu=\mu \max * \operatorname{RedI} * \operatorname{RedT} * \operatorname{RedP} * \operatorname{RedN}
$$

où RedI, RedT, RedN et RedP représentent respectivement la réduction par la lumière, la température, l'azote et le phosphore.

Lorsque les éléments nutritifs sont présents en excès, la croissance devient une fonction de la lumière et de la température (Morgan \& Kalff 1979) dont les effets écologiques sont inséparables, en raison de l'étroite relation entre le métabolistne et la saturation en lumière (Wetzel 1983).

$$
\begin{array}{r}
\mu=\mu \max * \text { RedI }{ }^{*} \operatorname{RedT} . \\
<- \text { terme } 1->
\end{array}
$$

\section{Matériel et méthodes}

\subsection{Choix des espèces}

Les espèces étudiées font partie des algues les plus représentatives de la biomasse lors des fleurs d'eaux observées entre 1983 et 1986 dans le lac d'Aydat (Alaoui \& Siriki 1985, Aleya \& El Magouri 1987) ; elles ont été isolées par Romagoux et Boumnich.

Il s'agit de :

- Fragilaria crotonensis Kitton

- Anabaena macrospara Klebahn

- Staurastrum pingue Teilling

- Coelastrum cambricum Archer

\subsection{Milieu de culture}

Le milieu de cult ure utilisé est celui proposé par Dauta (1982). Totalement minéral, il évite toute prolifération bactérienne et son équilibre chimique autorise des cultures de longue durée. Afin de prévenir un appauvrissement du milieu en dioxyde de carbone $\left(\mathrm{CO}_{2}\right)$, les cultures sont fortement bullées $(0.5 \mathrm{I}$. air.1-1 de culture. $\mathrm{mn}^{-1}$ ) avec de l'air comprimé fíltré (filtre de $1_{\mu} \mathrm{m}$ ) insufflé à la base des flacons.

Pour certaines espèces qui exigent la présence de matière organique pour se développer (Provasoli 1958), ce qui est le cas de Fragilaria crotonensis et Anabaena macrospora, le milieu est enrichi en vitamines (Thiamine $0.1 \mathrm{mg} . \mathrm{l}^{-1}$; Vit. $\mathrm{B}_{12} 0,5 \mu \mathrm{g}$. $\mathrm{l}^{-1}$ et Biotine $0.5 \mu \mathrm{g} . \mathrm{l}^{-1}$ ) et ajusté à un $\mathrm{pH}$ de 7,6 par de l'HCL dilué.
L'éclairement est donné selon un cycle de 15/9 ( $15 \mathrm{~h}$ de lumière pour $9 \mathrm{~h}$ d'obscurité) par des tubes fluorescents blancs (type cool-white) ou par des lampes PhytoClaude de 400 Watts. La mesure des lumières incidentes a êté rẹ́alisée à l'aide d'un quantamètre Li-Cor $185 \mathrm{~A}$.

\subsection{Protocole expérimental}

La souche mère utilisée est maintenue en phase exponentielle par des repiquages fréquents ( 2 à $3 \mathrm{j}$ ) dans un milieu riche en nutriments et dans des conditions héliothermiques suboptimales. Avant l'expérience destinée à la mesure du taux de croissance $(I, T)$, la culture est placée 24 heures à l'obscurité (synchronisation et non préadaptation à la lumière). Au début de l'expérience, la culture est largement diluée dans un milieu neuf pour éviter tout effet d'auto-ombrage.

Pour chaque température, la culture diluée est répartie dans une série de flacons (erlens de $300 \mathrm{ml}$ ) avec bullage sous un éclairage vertical, dont les intensités lumineuses sont comprises entre 5 et $800 \mu$ E.m-2.s- ${ }^{-1}$.

Le taux de croissance est déterminé à partir de la densité optique (mesuré à $750 \mathrm{~nm}$ dans une cuve de $5 \mathrm{~cm}$ ), représentative de la biomasse, mesurée au début du cycle lumineux $\left(t_{1}\right)$ et après $24 \mathrm{~h}$ d'incubation $\left(t_{2}\right)$. Le taux de croissance est calculé par la formule suivante:

$$
\mu=\left(t_{2}-t_{1}\right)-1 \operatorname{Ln}\left(A_{2} / A_{1}\right) .
$$

\section{Résultats}

Les figures 1 à 4 reportent les taux de croissance enregistrés à 6 températures $(10,15,20,25,30$ et $35^{\circ} \mathrm{C}$ ) et des lumières comprises entre 5 et $800 \mu$ E.m-2.s-1. La relation liant le taux de croissance à la lumière peut être décrite au moyen de plusieurs équations dont celles de Smith (1936), Steele (1962) ou Peeters \& Eilers (1978). Nous avons retenu cette dernière formule qui tient compte de l'effet inhibiteur des fortes intensités lumineuses sur la croissance algale.

La chlorophycée Coelastrum cambricum montre le taux de croissance le plus élevé $1,4 . j-1$ à une température de $35^{\circ} \mathrm{C}\left( \pm 1^{\circ} \mathrm{C}\right)$ et une intensité lumineuse de $290{ }_{\mu} \mathrm{E} \cdot \mathrm{m}^{-2}{ }^{2} \mathrm{~s}^{-1}$, valeur très proche de celle obtenue par Dauta (1982) sur une espèce voisine : Coelastrum microsporum. Le plus faible taux de croissance est obtenu avec Fragilaria crotonensis 
0,57 à une température de $25^{\circ} \mathrm{C}$ et une intensité lumineuse de $150{ }_{\mu} \mathrm{E} \cdot \mathrm{m}^{-2} \cdot \mathrm{s}^{-1}$, valeur légèrement supérieure à celle obtenue par Hartig \& Wallen (1986) sur la même espèce $\left(0,40 . j-1\right.$ à $23^{\circ} \mathrm{C}$ ). A notre connaissance, pour les deux autres espèces (Anabaena macrospora et Staurastrum pingue), aucune étude relative à leur croissance n'a été faite.

Pour chaque température, il est possible de définir un taux de croissance optimal $\left(\mu \max _{\mathrm{T}}\right)$ et une intensité lumineuse optimale associée ( $\operatorname{lopt}_{T}$ ). Les variations de $\mu \max _{\mathrm{T}}$ et de Iopt $\mathrm{T}$ peuvent être reportées en fonction de la température (fig. 5 et 6) par un ajustement du modèle proposé par Lehman et al. (1975).

\section{Discussion}

Malgré le nombre de travaux consacrés à l'écologie du phytoplancton, l'interdépendance entre la lumière et la température reste encore mal quantifiée.

Selon Schnoor \& Di Toro (1980), les chlorophycées produisent les plus forts taux de croissance bruts 1 à $1,75, j-1$. Les plus faibles taux bruts de croissance (inférieurs à $0,5 . \mathrm{j}^{-1}$ ) appartiennent aux Cyanophycées et aux Flagellés. Tandis que les Diatomées ont une position intermédiaire avec des taux de croissance compris entre 0,25 et $0,75 . j^{-1}$.
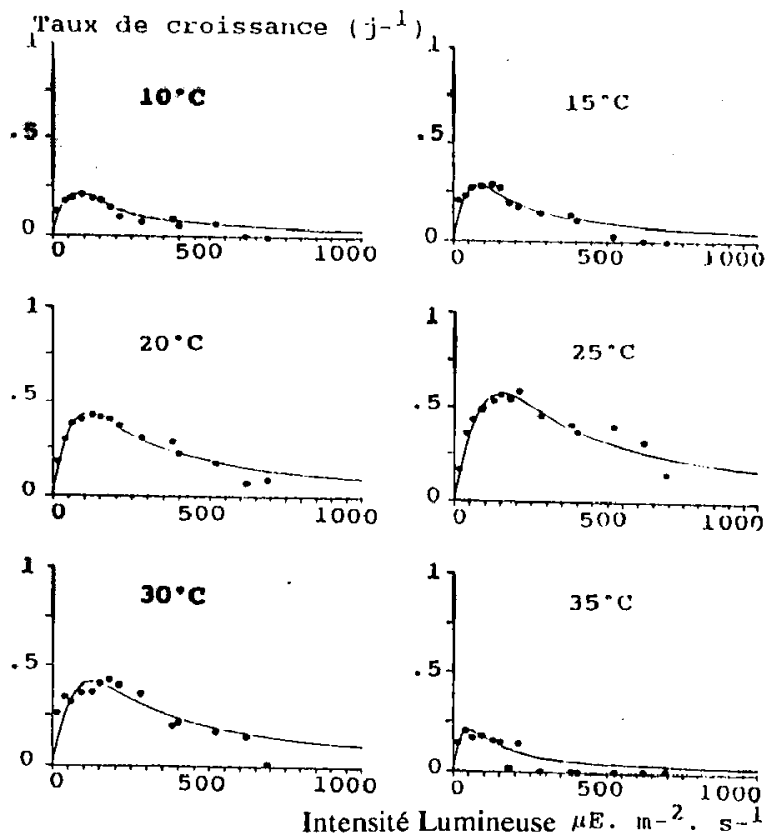

Fig. 1. Fragilaria crotonensis : Interaction de la lumière et de la température sur le taux de croissance.

(Ajustement graphique par le modèle de Peeters \& Eilers 1978). 

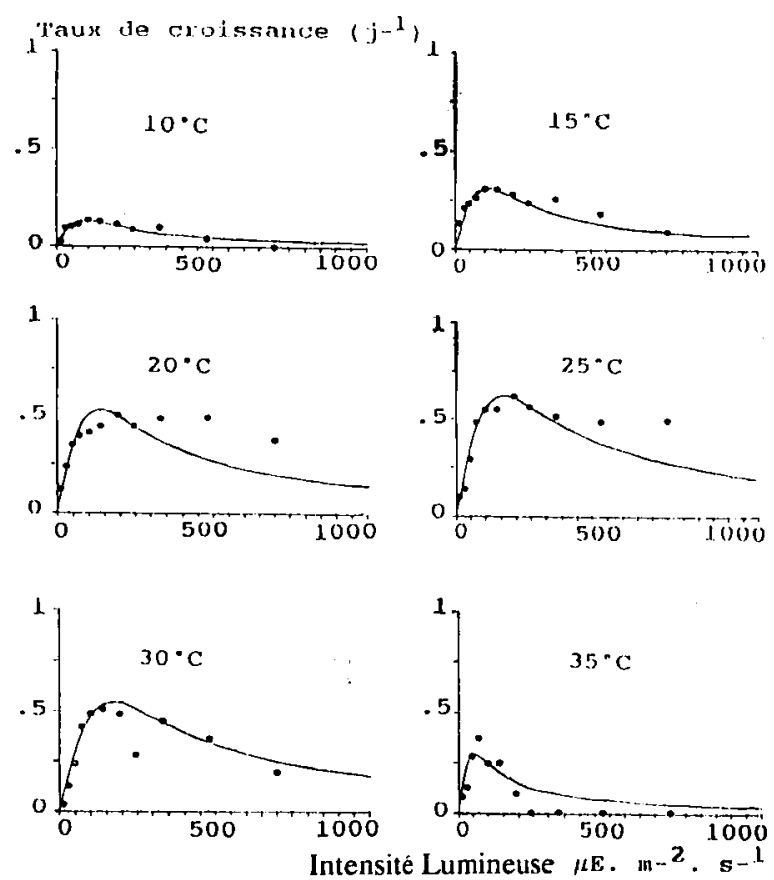

Fig. 2. Anabaena macrospora: Interaction de la lumière et de la température sur le taux de croissance. (Ajustement graphique par lé modèle de Peeters \& Eilers 1978).

Grand nombre d'auteurs ont observé que les températures optimales des Chlorophycees sont le plus souvent supérieures à $30^{\circ} \mathrm{C}$ (Felfoldy 1962, Aruga 1965, Gargas 1978), et celles des Cyanophycées comprises entre 27 et $35^{\circ} \mathrm{C}$ (Krueger \& Eloff 1978). L'optimum est plus bas pour les Diatomées (Eppley 1977, Dauta 1982, Hartig \& Wallen 1986). Les valeurs des optimurns thermiques deduites de nos expériences corroborent les observations de ces auteurs sauf pour Anabaena macrospora où l'optimum thermique est de $25^{\circ} \mathrm{C}$.

Les variations des intensités lumineuses optimales, ont été déjà précisées par divers auteurs tels
Sorokin \& Krauss (1962), Smayda (1969), Cloern (1976) et Eppley (1977). L'importante variation de celle-ci est attribuée à l'adaptation de certaines espèces d'algues à diverses lumières (Jorgensen 1969 , Raps et al. 1983). D'une façon générale, les diatomées et les cyanophycées répondent à des intensités lumineuses beaucoup plus faibles que celles des chlorophycées.

Les résultats présentés ici, variations du $\mu \max _{\mathrm{T}}$, sont en accord avec les observations expérimentales de Post et al. (1985) et apportent des valeurs de constantes spécifiques utiles à la comprẻhension des successions phytoplanctoniques dans les milieux 

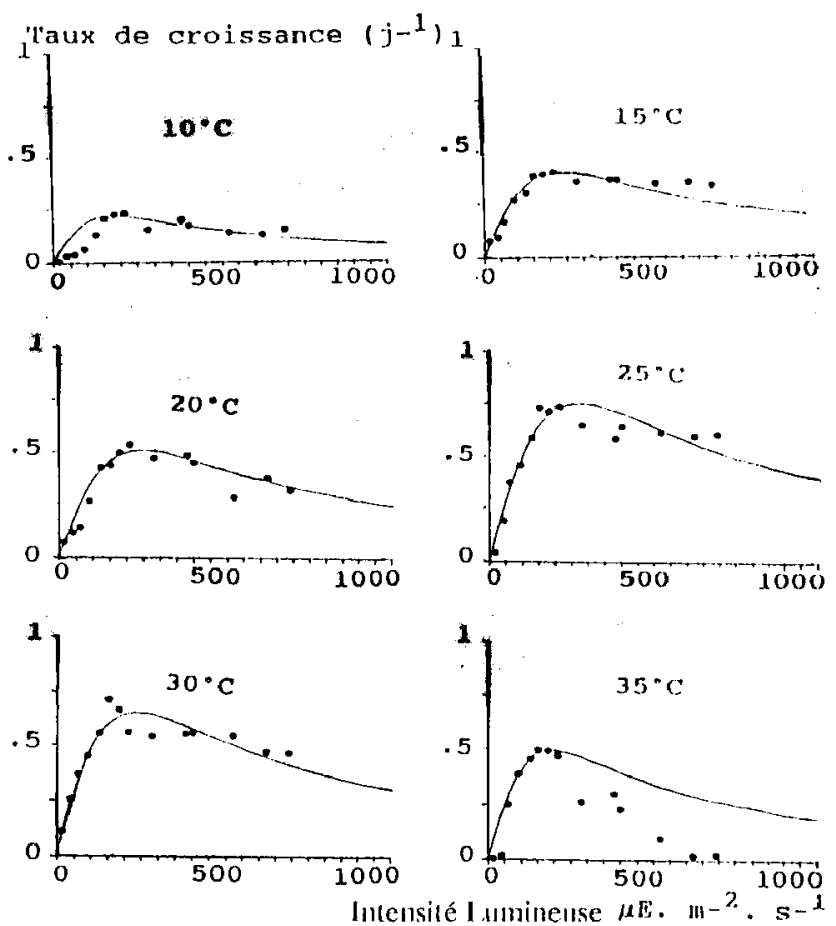

Fig. 3. Staurastrum pingue : Interaction de la lumière et de la température sur le taux de croissance. (Ajustement graphique par le modèle de Peeters \& Eilers 1978). 

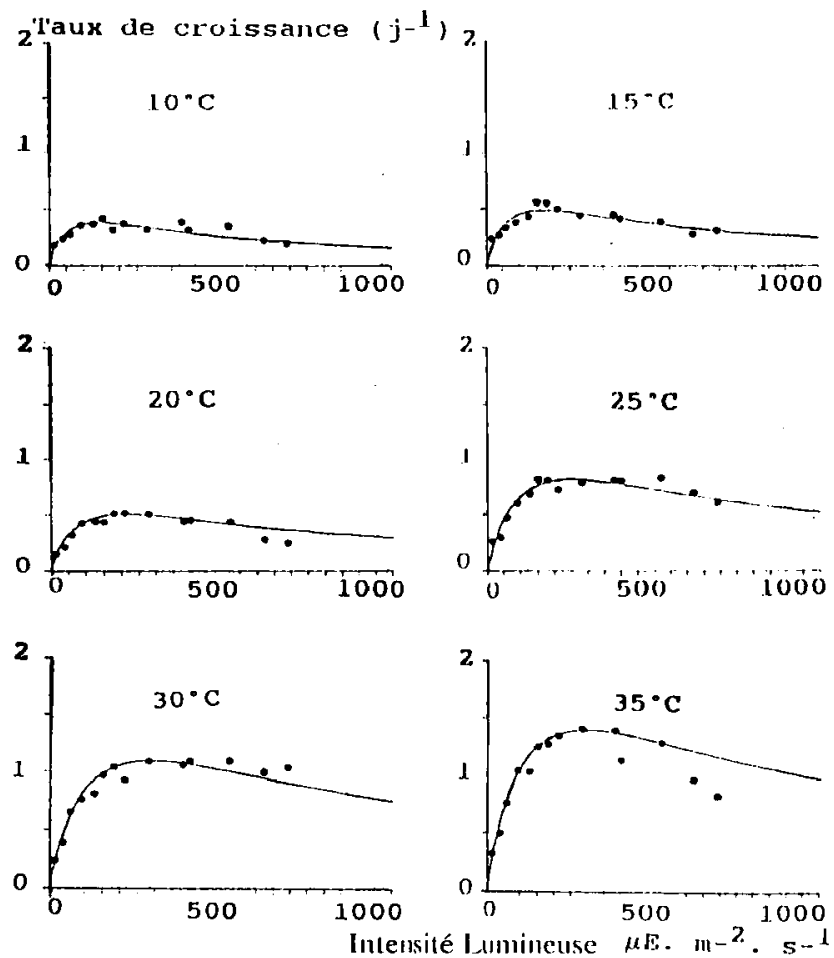

Fig. 4. Coelastrum cambricum: Interaction de la lumière et de la température sur le taux de croissance. (Ajustement graphique par le modèle de Peeters \& Eilers 1978). 

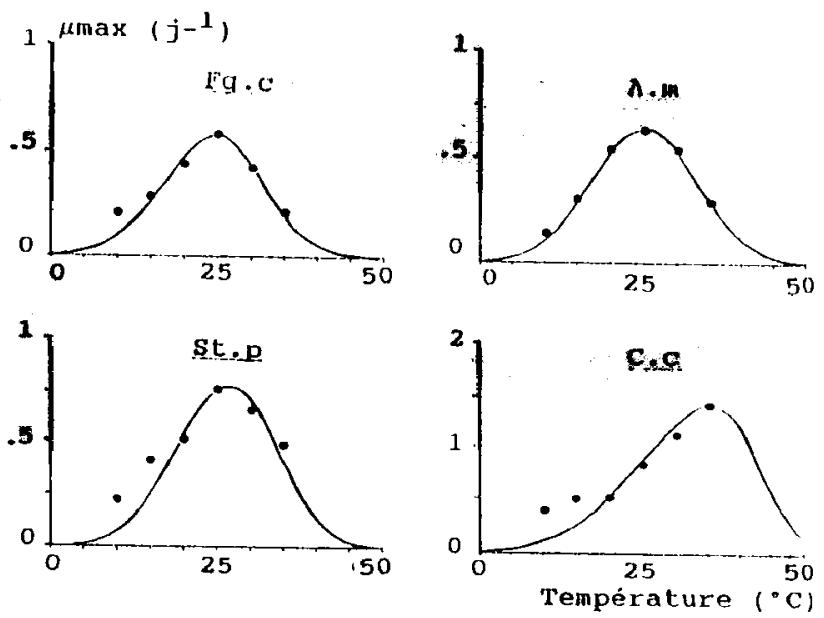

Fig. 5. Variation de $\mu$ max en fonction de la têmpérature pour Fragilaria crotonensis ( $\mathrm{Fg.c}$ ), Anabaena macrospora (A.m), Staurastrum pingue (St.p) et Coelastrum cambricum (C.c.). (Ajustement graphique au modèle de Lehrnan et al. 1975).
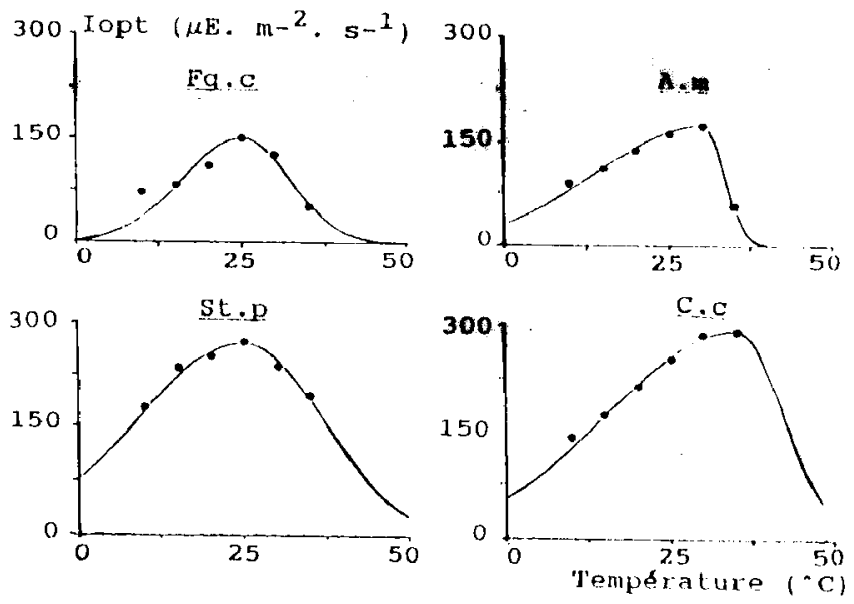

Fig. 6. Variation de Iopt en fonction de la température pour Fragilaria crotonensis (Fg.c), Anabaena macrospora (A.m), Staurastrum pingue (St.p) et Coelastrum cambricum (C.c). (Ajustement graphique au modèle de Lehman et al. 1975). 
naturels. Toutefois, ces résultats obtenus en conditions nutritionnelles non limitantes seront complétés par des études relatives à l'assimilation et au stockage des principaux éléments nutritifs (N, P, Si) afin d'avoir un ensemble de données permettant de déterminer les conditions de compétition de ces espèces dans les milieux naturels.

\section{Travaux cilés}

Alaoui (M.) \& Siriki (D.). 1985. - Dynamique des populations et évolution métabolique du phytoplancton d'un lac eutro. phe (Lac d'Aydat, Puy de Dôme, France). Thèse Doct. 3e cycle. Univ. Clermont II, 206 p.

Aleya (L.) \& El Magouri (H.). 1987. - Dynamique des populations phyroplanctoniques du lac d'Aydat, Puy de Dôme (France). Biomasse et activité métabolique de diverses fractions de taille. Thèse Doct. $3^{\mathrm{c}}$ cycle. Univ. Clermont II, $216 \mathrm{p}$.

Aruga (Y.). 1965. Ecological studies of photosynthesis. II photosynthesis of algae. Bot. Mg. Tokio., $78: 360-365$.

Bierman (V.J.). 1976. - Mathernatical model of the selective enhancement of blue-green algae by nutriment enrichement. In Canale (R.P.) : Modelling Biochemical Processes in Aquatic Ecosystems. Ann Arbor Sciences Pub. : 1-31.

Canale (R.P.), De Palma (L.M.) \& Vogel (A.). 1976. - A plankton-based food web model for lake Michigan. In Canale (R.P.) : Modelling Biochemical Processes in aquatic Ecosystems. Ann Arbor Sciences Publ. : 33-74.

Cloern (J.E.). 1976. - Population dynamics of Cryptomonas ovata. A laboratory, field and computer study. Thèse P.H.D. Washington State Univ. : 102 p.

Daut a (A.). 1982. - Conditions de développement du phytoplancton. Etude comparative du comportement de huit especes en culture. 1. Détermination des paramètres de croissance en fonction de la lumière et de la température. Ann/s $\mathrm{Lim}$ nol, 18 (3) : 217-262.

Eppley (R.W.) 1977. - The growth and culture of diatoms. In (D.) Werner (Ed.) : The Biology of Diatoms, Botanical monographs, $13: 24-64$.

Felfoldy (L.J.M.). 1962. - On the role of $\mathrm{pH}$ and inorganic car* bon sources in photosynthesis in unicellular algae. Acta Biol. Acad. Sci. Hung., $13: 297-314$

Gargas (E.). 1978. - Phytoplancton production chlorophyll-a and nutrients in the Open Danish Waters 1975-1977. The Belt Project. The national Agency of environmental Protection, Denmark, $61 \mathrm{p}$.
Hartig (H.) \& Wallen (G.). 1986. - The influence of light and temperature on growth and photosynthesis of Fragilario crotonensis Kinon. Journal of Freshwater Ecology, 3 (3) : $371+382$

Jorgensen (E.G.). 1969. - The adaptation of plankton algae. IV. Light adaptation in different algal species. Physiol. Plam, 22 (6) : $1307-1315$.

Krueger (G.H.J.) \& Eloff (J.N.). 1978. - The effect of temperature on specific growth rate and activation energy of Microcystis and Synechococcus isolates relevant to the onset of natural blooms. J. Limnol. Soc. Sth. Afr., 4 (1) : 9-20.

Lehman (J.T.), Botkin (D.B.) \& Likens (G.E.). 1975. - The assumptions and rationales of a computer model of phytoplankton population dynamics. Limmol. Oceanogr., 20 (3) : 343-364.

Morgan (K.C.) \& Kalff (J.). 1979. - Effect of light and temperature interactions on growth of Cryptomonos erosa (Cryptophyceae). J. Phycol., $15: 127-134$.

Peeters (J.C.H.) \& Eilers (P.). 1978, - The relationship between light intensity and photosynthesis a simple mathematical model, Hydrobiol. Bull., 12 : 134-136.

Post (A.F.), (R.) de Witt \& (L.R.) Mur. 1985. - Interactions between temperature and light intensity on growth and photosynthesis of the cyanobacterium Oscillatoria agardhii. J. Plancton Res. 7 : $\mathbf{4 8 7 . 4 9 5}$.

Provasoli (L.). 1958, - Nutrition and Ecology of Protozoa and Algae. Ann. Rev. Microbiol, 12:297-308.

Raps (S.), Wyman (K.), Siegelman (H.W.) \& Falkowski (P.G.). 1983. - Adaptation of the Cyanobacterium Microcystis aemiginosa to light intensity. Plant Physiol, 72 : 829-832.

Reynolds (C.S.). 1984 - The ecology of freshwater phytoptanc Ion. Cambridge University press, London : $384 \mathrm{p}$.

Schnoor (J.L.) \& Di Toro (D.). 1980. - Differential phytoplankton sinking and growh rates : An eigenvalue analysis. Ecol. Modelling. 9 : 233-245.

Smayda (T.J.). 1969. - Experimental observations on the influence of temperature, light and salinity on cell division of the marine diatom Detonula confervacea (Cleve). Gran. J. Phycol., 5 : 105-157.

Smith (E.L.). 1936. - Photosynthesis in relation to light and carbon dioxyde. Proc. Nat. Acad. Sc. Wash., 22 : 504-511.

Sorokin (C.) \& Krauss (R.W.). 1962. - Effects of temperature and illuminance on chlorella growth uncoupled from cell division. Plant Physiol., $37: 37-42$.

Wetzel (R.G.). 1983. - Limnology. W,B. Saunders Campany, (Philadelphia), 767 p. 